\title{
SUBALGEBRAS OF SPACES OF CONTINUOUS FUNCTIONS
}

\section{WALTER RUDIN}

I. Introduction. Let $\mathfrak{e}(X)$ be the set of all complex-valued continuous functions on the compact Hausdorff space $X ; \mathrm{e}(X)$ is a Banach algebra if the norm is defined by $\|f\|=\sup |f(x)|(x \in X)$. If $f_{1}, \cdots, f_{n}$ are members of $\mathfrak{e}(X), \mathfrak{R}\left(f_{1}, \cdots, f_{n}\right)$ denotes the smallest closed subalgebra of $\mathfrak{e}(X)$ which contains $f_{1}, \cdots, f_{n}$, and the complex constants. As usual, a family $F \subset C C(X)$ is said to separate points on $X$ if for each pair $x_{1}, x_{2} \in X\left(x_{1} \neq x_{2}\right)$ there is some $f \in \mathcal{F}$ such that $f\left(x_{1}\right) \neq f\left(x_{2}\right)$.

The purpose of this paper is twofold. First, Wermer has shown that $\mathfrak{e}(X)$ contains maximal closed subalgebras (which separate points and are not maximal ideals) if $X$ is a simple closed curve, and we now prove that $\mathfrak{e}(X)$ contains such subalgebras whenever the Cantor set can be topologically embedded in $X$ (Theorem 2); if $X$ is a simple closed curve, the algebra described in Theorem 2 is quite different from those found by Wermer $[5 ; 6]$.

Secondly, if $I$ is the unit interval and $f \in \mathbb{C}(I)$ is one-to-one on $I$, it is well known that $R(f)=\mathfrak{C}(I)$ [4, p. 444]. Turning to rings with two generators, let $f, g \in \mathfrak{C}(I)$, and suppose $R(f, g)$ separates points on $I$. By the Stone-Weierstrass theorem, $\mathfrak{R}(f, g)=\mathfrak{e}(I)$ if both $f$ and $g$ are real. We show that $\mathcal{R}(f, g)=\mathfrak{C}(I)$ even if only one of the two generating functions is real (this is merely a special case of Theorem 3 ), but that the conclusion is false if neither $f$ nor $g$ are required to be real (Theorem 5). This solves a problem posed by Mergelyan [3, p. 29], but immediately raises a new one, still unsolved: what conditions (like differentiability, bounded variation, etc.) imposed on $f$ and $g$ will assure that $\mathbb{R}(f, g)=\mathfrak{C}(I)$ even if neither of the functions is real?

Note that the mapping

$$
s \rightarrow(f(s), g(s))
$$

is a homeomorphism onto an $\operatorname{arc} L$ embedded in the space of two variables (real or complex, as the case may be), and that $R(f, g)=\mathcal{C}(I)$ if and only if every continuous function on $L$ can be approximated uniformly by polynomials in these two variables (with complex coefficients). We are thus led to problems concerning uniform ap-

Presented to the Society, April 16, 1955; received by the editors May 10, 1955 and, in revised form, September 26, 1955. 
proximation by polynomials in several variables, and in particular to Theorem 4, which is an extension of a theorem of Mergelyan [3, p. 18] (which states that if $f \in \mathcal{C}(E)$, where $E$ is a compact set in the $z$-plane with connected complement, and if $f$ is analytic at every interior point of $E$, then $f$ can be approximated uniformly on $E$ by polynomials in $z$ ).

The reason for combining these results in one paper lies in the fact that a certain algebra of dimension-raising mappings was found to be useful in their proofs, and we begin by describing this algebra.

II. An algebra of dimension raising mappings. Let $E$ be a totally disconnected bounded perfect set in the plane, such that the intersection of $E$ with any open set is either empty or has positive twodimensional Lebesgue measure. Let $S$ be the Riemann sphere (the plane plus the point at infinity) and let $a$ be the subalgebra of $\mathfrak{e}(S)$ consisting of all $f \in \mathrm{e}(S)$ which are analytic in $S-E$.

TheOREM 1. (a) Given $z_{0} \in E, \epsilon>0, \eta>0$, there exists a function $p \in a$ such that $\left|z-z_{0}\right| \geqq \eta$ implies $\left|p(z)-\left(z-z_{0}\right)^{-1}\right| \leqq \epsilon$.

(b) For every $f \in a, f(E)=f(S)$.

(c) a contains a set of three functions which separates points on $S$.

Proof. Put

$$
q(z)=\iint_{z} \frac{d s d t}{z-u} \quad(u=s+i t)
$$

Since $z q(z) \rightarrow m(E)>0$ as $z \rightarrow \infty, q$ is not constant; $q$ is evidently analytic in $S-E$; to show that $q \in \mathcal{C}(S)$, suppose $\left|z_{1}-z_{2}\right|=\delta<1$, let $2 z_{3}=z_{1}+z_{2}$, and divide the integral defining $q\left(z_{1}\right)-q\left(z_{2}\right)$ into three parts, corresponding to the intersections of $E$ with (1) $\left|u-z_{3}\right| \leqq \delta$, (2) $\delta<\left|u-z_{3}\right| \leqq 1$, (3) $1<\left|u-z_{3}\right|$. Easy estimates of these integrals show that the major contribution is furnished by (2) and that $q$ has a modulus of continuity of the form $C \delta \log (1 / \delta)$, a result stated (without proof) in [2]. Hence $a$ contains nonconstant functions.

To prove (a), let $E_{r}=\left\{z \in E|| z-z_{0} \mid \leqq r\right\}$ and define

$$
p_{r}(z)=\frac{1}{m\left(E_{r}\right)} \iint_{E_{r}} \frac{d s d t}{z-u} \quad(u=s+i t) .
$$

It is clear that $\lim _{r \rightarrow 0} p_{r}(z)=\left(z-z_{0}\right)^{-1}$, uniformly in $\left|z-z_{0}\right| \geqq \eta$.

We prove (b) in accordance with a written communication from J. Wermer: it is enough to show that if $f(S)$ contains the origin, then $f(z)=0$ for some $z \in E$. If $f(z) \neq 0$ on $E$, then $f$ has only a finite number of zeros in the finite plane, say at $z_{1}, \cdots, z_{k}$ (counted with their multiplicities), and the function 


$$
g(z)=f(z) \prod_{i=1}^{k}\left(z-z_{i}\right)^{-1}
$$

is in $a$. Since $g$ has no finite zeros, a single-valued branch of $\log g(z)$ can be defined in the finite plane; but this contradicts the analyticity of $g$ and the fact that $g(\infty)=0$.

To prove (c), choose a point $a \in S-E$ such that $q(a) \neq 0$ (where $q$ denotes the same function as above), and put $q_{1}(z)=q(z), q_{2}(z)$ $=z q(z), q_{3}(z)=(q(z)-q(a)) /(z-a)$. Suppose $z_{1} \neq z_{2}$ and $q_{1}\left(z_{1}\right)=q_{1}\left(z_{2}\right)$ $=b$; if $b \neq 0$ then $q_{2}\left(z_{1}\right) \neq q_{2}\left(z_{2}\right)$; if $b=0$, then $q_{3}\left(z_{1}\right) \neq q_{3}\left(z_{2}\right)$. The theorem follows.

Part (b) shows that every nonconstant $f \in Q$ maps the zero-dimensional set $E$ onto a set of dimension 2 (actually, onto the closure of an open set). With regard to (c), it is not known whether some two functions in a separate points, even on $E$.

\section{Maximal subalgebras of $\mathfrak{e}(X)$.}

THEOREM 2. If $X$ is a compact Hausdorff space which contains a subset $K$ homeomorphic to the Cantor set, ${ }^{1}$ then $\mathfrak{C}(X)$ contains a closed subalgebra $\mathrm{NC}$ with the following properties:

(i) $\mathfrak{T}$ separates points on $X$.

(ii) $\mathfrak{T l}$ is a maximal subalgebra of $\mathfrak{C}(X)$; i.e., $\mathfrak{N}$ is not a proper subset of any closed proper subalgebra of $\mathfrak{e}(X)$.

(iii) If $f=u+i v$, where $u$ and $v$ are real, and $f \in \Re$, then $u(K)$ is connected.

If $h$ is a homeomorphism of $K$ onto the Cantor set, then $h$ can be extended to a continuous real function on $X$ (since compact Hausdorff spaces are normal) which, by (iii), is not in $\Re$. Consequently $\mathfrak{T} \neq \mathfrak{e}(X)$.

Proof. Let $E$ be as in Theorem 1, and form the following algebra $a_{E} \subset \mathfrak{C}(E): f \in Q_{E}$ if and only if there is a function $g \in Q$ such that $f(z)=g(z)$ on $E$. By the maximum modulus theorem, $a_{E}$ is closed. It will be shown that $Q_{E}$ is contained in a maximal closed subalgebra of $\mathfrak{C}(E)$; whether $a_{E}$ is itself maximal is an open question.

By Zorn's lemma there exists a closed algebra $B \subset \mathfrak{C}(E)$ which is maximal with the respect to the following properties: (1) $a_{E} \subset \mathbb{B}$; (2) if $f \in B$ and $f=u+i v(u, v$ real), then $u(E)$ is connected. Since $E$ is homeomorphic to the Cantor set, $B \neq \mathfrak{C}(E)$.

${ }^{1}$ An example of an uncountable compact Hausdorff space which contains no copy of the Cantor set is furnished by the set of all ordinals less than or equal to the first uncountable, with the usual order topology. It is well known that every separable metric compact space which is totally disconnected and perfect is homeomorphic to the Cantor set. 
Suppose now that $B \subset B^{\prime} \subset \mathcal{C}(E)$, where $B \neq B^{\prime}$ and $B^{\prime}$ is a closed algebra. The choice of $B$ shows that there is a function $f \in B^{\prime}, f=u+i v$ such that $u(z)>0$ on $E_{1}, u(z)<0$ on $E_{2}$, where $E_{1}$ and $E_{2}$ are nonempty closed subsets of $E$, and $E=E_{1} \cup E_{2}$. Covering $f\left(E_{1}\right)$ and $f\left(E_{2}\right)$ by disjoint closed simply connected regions, we see via the Mergelyan theorem $\left[3\right.$, p. 18] that there is a sequence of polynomials $P_{n}$ such that $P_{n}(w) \rightarrow 1$ if $w \in f\left(E_{1}\right)$ and $P_{n}(w) \rightarrow 0$ if $w \in f\left(E_{2}\right)$, uniformly. Since $B^{\prime}$ is closed, the function

$$
g(z)=\lim _{n \rightarrow \infty} P_{n}(f(z))= \begin{cases}1 & \left(z \in E_{1}\right), \\ 0 & \left(z \in E_{2}\right)\end{cases}
$$

belongs to $\mathrm{B}^{\prime}$.

Choose $h \in \mathfrak{C}(E)$. If $z_{0} \in E_{2}$, Mergelyan's theorem ${ }^{2}[3$, p. 18] shows that $h$ can be approximated uniformly on $E_{1}$ by polynomials in $\left(z-z_{0}\right)^{-1}$, and we infer from Theorem 1(a) that there is a sequence of functions $\phi_{n} \in Q_{E}$ which converges to $h$ uniformly on $E_{1}$. Similarly, there are functions $\psi_{n} \in Q_{E}$ which converge to $h$ uniformly on $E_{2}$. Combining these facts, we obtain

$$
\lim _{n \rightarrow \infty}\left\{g(z) \phi_{n}(z)+[1-g(z)] \psi_{n}(z)\right\}=h(z)
$$

uniformly on $E$. Hence $h \in B^{\prime}, \mathbb{B}^{\prime}=\mathfrak{C}(E)$, and $B$ is a maximal closed subalgebra of $\mathfrak{e}(E)$ which, by Theorem 1 (c), separates points on $E$.

Now let $X$ and $K$ satisfy the hypotheses of Theorem 2 ; since $K$ and $E$ are homeomorphic, the continuous functions on $E$ can be transferred to $K$, and $B$ gives rise to a maximal closed subalgebra $B_{1} \subset \mathfrak{C}(K)$. Let $\mathfrak{T}$ be the set of all $f \in \mathfrak{C}(X)$ whose restrictions to $K$ are members of $B_{1}$. It is obvious that $\mathfrak{T}$ satisfies (i) and (iii); to establish (ii), suppose $\mathfrak{M} \subset \mathfrak{T}^{\prime} \subset \mathfrak{C}(X)$, where $\mathfrak{T} \neq \mathfrak{M}^{\prime}$ and $\mathfrak{T}^{\prime}$ is a closed algebra.

If $\phi \in \mathfrak{C}(X)$ and $\epsilon>0$, there is some $f \in \mathfrak{M}^{\prime}$ such that $|f(x)-\phi(x)|<\epsilon$ on $K$. Since $X$ is normal, there is some $g \in \mathfrak{e}(X)$ such that $g(x)$ $=f(x)-\phi(x)$ on $K$ and $|g(x)|<\epsilon$ on $X$. Put $h=g+\phi$. Then $h(x)-f(x)$ $=0$ on $K$, so that $h-f \in \mathfrak{N}$, and $h \in \mathfrak{N}^{\prime}$. Also, $|h(x)-\phi(x)|<\epsilon$ on $X$, which proves that $\mathfrak{T}^{\prime}=\mathfrak{C}(X)$. The theorem follows.

\section{Uniform approximation by polynomials.}

Theorem 3. Suppose $f, g_{1}, \cdots, g_{n} \in \mathbb{C}(I)$, where $I$ is the unit interval, and $g_{1}, \cdots, g_{n}$ are real. If $R\left(f, g_{1}, \cdots, g_{n}\right)$ separates points on $I$, then $\mathfrak{R}\left(f, g_{1}, \cdots, g_{n}\right)=\mathfrak{e}(I)$.

2 Actually, an earlier theorem of Lavrent'ev would suffice here (see the bibliography in [3]). 
The remark made in the introduction shows that Theorem 3 is equivalent to the following proposition: if $L$ is an arc in the Euclidean space $R^{n+2}$ of the variables $\left(z, t_{1}, \cdots, t_{n}\right)$, where $z$ is complex and $t_{1}, \cdots, t_{n}$ are real, then every member of $\mathfrak{e}(L)$ is the uniform limit of a sequence of polynomials in $\left(z, t_{1}, \cdots, t_{n}\right)$.

This proposition is a special case of the following theorem, where we put $\left(t_{1}, \cdots, t_{n}\right)=t$ :

THEOREM 4. Let $K$ be a compact set in $R^{n+2}$, such that the set $K_{t}$ $=\{z \mid(z, t) \in K\}$ does not separate the z-plane for any $t$. Suppose $f \in \mathfrak{e}(K)$, and put

$$
f_{t}(z)=f(z, t) \quad\left(z \in K_{t}\right) .
$$

If, for every $t, f_{t}$ is analytic at every interior point of $K_{t}$, then $f$ can be approximated, uniformly on $K$, by polynomials in $\left(z, t_{1}, \cdots, t_{n}\right)$.

Proof. Let $\epsilon>0$ be given. Let $T$ be the set of all $t$ such that $(z, t)$ $\in K$ for some $z$. For each $t \in T$ there exists, by Mergelyan's theorem, a polynomial $P_{t}$ such that

$$
\left|P_{t}(z)-f_{t}(z)\right|<\epsilon \quad\left(z \in K_{t}\right) .
$$

The compactness of $K$ and the uniform continuity of $f$ imply that each $t \in T$ has a neighborhood $N_{t} \subset R^{n}$ such that

$$
\left|P_{t}(z)-f(z, s)\right|<\epsilon \quad\left(s \in N_{t},(z, s) \in K\right) .
$$

The sets $N_{t}$ form an open covering of the compact set $T$ from which a finite subcovering can be extracted. Thus there exist polynomials $Q_{i}$ and neighborhoods $V_{i} \subset R^{n}(1 \leqq i \leqq m)$ whose union contains $T$, such that

$$
\left|Q_{i}(z)-f(z, t)\right|<\epsilon \quad\left(t \in V_{i},(z, t) \in K\right) .
$$

Now let $\alpha_{i}$ be a continuous real function, positive in $V_{i}$, zero in $R^{n}-V_{i}$, and put

$$
\beta_{i}(t)=\frac{\alpha_{i}(t)}{\alpha_{1}(t)+\cdots+\alpha_{m}(t)} \quad(1 \leqq i \leqq m, t \in T) .
$$

The choice of $Q_{i}$ and $\beta_{i}$ shows that

$$
\beta_{i}(t)\left|Q_{i}(z)-f(z, t)\right| \leqq \epsilon \beta_{i}(t) \quad(1 \leqq i \leqq m,(z, t) \in K) .
$$

Since $\beta_{1}(t)+\cdots+\beta_{m}(t)=1$ for $t \in T$, we have

$$
\left|f(z, t)-\sum_{i=1}^{m} \beta_{i}(t) Q_{i}(t)\right|=\left|\sum_{i=1}^{m} \beta_{i}(t)\left\{f(z, t)-Q_{i}(z)\right\}\right| \leqq \epsilon
$$


for all $(z, t) \in K$. But the functions $\beta_{i}$ can be approximated uniformly on $T$ by polynomials in $\left(t_{1}, \cdots, t_{n}\right)$, by the Stone-Weierstrass theorem, and the proof is complete.

Theorem 5. Given a totally disconnected perfect subset $K$ of the unit interval $I$, there exists a closed algebra $R(f, g) \subset \mathfrak{C}(I)$ which separates points on $I$, such that $h(K)$ is the closure of an open subset of the plane, for every nonconstant $h \in \mathbb{R}(f, g)$. In particular, $\mathbb{R}(f, g) \neq \mathfrak{C}(I)$.

Proof. Let $E$ and $a$ be as in Theorem 1. Choose a nonconstant $q_{1} \in Q$ such that $q_{1}(\infty)=0$, put $q_{2}(z)=z q_{1}(z)$, so that $q_{2} \in Q$, and let $E_{0}=\left\{z \in E \mid q_{1}(z)=0\right\}$. The mapping

$$
z \rightarrow\left(q_{1}(z), q_{2}(z)\right)
$$

is one-to-one on $E-E_{0}$, so that $E$ is mapped onto a totally disconnected bounded perfect set $E^{*}$ in the space $R^{4}$ of two complex variables $\left(w_{1}, w_{2}\right)$.

If $P\left(w_{1}, w_{2}\right)$ is a polynomial, then $P\left(q_{1}, q_{2}\right) \in a$, and Theorem 1 (b) shows that $\phi\left(E^{*}\right)$ is the closure of a plane open set for every nonconstant $\phi \in \mathfrak{C}\left(E^{*}\right)$ which is the uniform limit of polynomials in $\left(w_{1}, w_{2}\right)$. Since $E^{*}$ is homeomorphic to the Cantor set, this implies (in contrast with Theorem 4$)$ that not every $\phi \in \mathfrak{C}\left(E^{*}\right)$ is a uniform limit of polynomials in $\left(w_{1}, w_{2}\right)$.

There exists an $\operatorname{arc} L$ in $R^{4}$ which contains $E^{*}[1$, p. 302], and we can choose two functions $f, g \in \mathcal{C}(I)$ such that the mapping $s \rightarrow(f(s)$, $g(s))$ is a homeomorphism of $I$ onto $L$ which carries $K$ onto $E^{*}$. Then $R(f, g)$ has the desired properties.

\section{REFERENCES}

1. Louis Antoine, Sur l'homémorphie de deux figures et de leurs voisinages, J. Math. Pures Appl. (8) vol. 4 (1921) pp. 221-325.

2. Arnaud Denjoy, Sur les fonctions analytiques uniformes qui restent continues sur un ensemble parfait discontinu de singularites, C. R. Acad. Sci. Paris vol. 148 (1909) pp. 1154-1156.

3. S. N. Mergelyan, Uniform approximations to functions of a complex variable, Uspehi Mathematičeskih (N.S.) vol. 7 (48) (1952) pp. 31-122; Amer. Math. Soc. Translations, no. 101, 1954.

4. J. L. Walsh, Über die Entwicklung einer Funktion einer komplexen Veränderlichen nach Polynomen, Math. Ann. vol. 96 (1926) pp. 437-450.

5. John Wermer, On algebras of continuous functions, Proc. Amer. Math. Soc. vol. 4 (1953) pp. 866-869.

6. - Bull. Amer. Math. Soc. vol. 61 (1955) p. 48.

UNIVERSITY OF ROCHESTER 